\title{
Hay espacio para el cambio en la educación: Perspectivas y debates, de educación critica a educación sustentable ${ }^{1}$
}

Is there space for change in Education: Perspectives and debates from critical literacy to sustainable education.

Juan Fernando Larco Guevara (Univeristy of Freiburg) (Master in Global Studies)

jfernando92@icloud.com

\section{Resumen}

El presente trabajo explora el concepto de educación y al mismo tiempo se vincula con la reconceptualización del mismo. El proposito principal es navegar en los diferentes cambios de como la educación es conceptualizada, entendida y aplicada en diferentes niveles. Los conceptos en torno a la educación y la manera en la que es percibida quizas hayan cambiado pero existe la necesidad de hacer un analisis más profundo en cuanto a las estructuras y su rol, para poder visualizar la posibilidad de un cambio en mayor beneficio de la educación y de un canal de comunicación abierto.

\begin{abstract}
The present work explores the concept of education and engages at the same time with the re-conceptualization of it. The main purpose is to navigate in the different shifts of how education is conceptualized, understood and applied at different levels. The concepts around education and the way is perceived may have changed but there is the need of doing a deeper analysis around the role and the structures, to sightsee the possibility of greater change in pro of education and an open education dialogue.
\end{abstract}

${ }^{1}$ Recibido: 28/08/2018 Evaluado: 01/09/2018 Aceptado: 06/09/2018 
Palabras claves: Giro educacional, Agenda global, Estudios post-coloniales, re-construcción del conocimiento, dialogo multicultural, debate educacional, debate estructural.

Keywords: Education Shift, Global Agenda, Post-colonial studies, re-construction of knowledge, multicultural dialogue, educational debate, structural debate.

\section{Planteamiento de la cuestión}

A teacher who establishes rapport with the taught, becomes one with them, learns more from them than he teaches them. He who learns nothing from his disciples is, in my opinion, worthless. Whenever I talk with someone I learn from him. I take from him more than I give him. In this way, a true teacher regards himself as a student of his students. If you will teach your pupils with this attitude, you will benefit much from them.

\section{Mahatma Ghandi}

En el cambio de siglo muchas cosas han sido discutidas en torno al tema de educación, esto demuestra que existe una necesidad de traer este tema no solo a nivel local o nacional pero sino también a la esfera internacional. Hoy en día se ha convertido en moda el hablar sobre el rol de la educación y la importancia de la misma en la agenda global.

Tomando en cuenta que la UNESCO establece que la educación es un derecho humano que posee tres componentes cobertura, acceso y calidad el resultado de esto es evidente. El compromiso de cumplir la agenda de educación 2030 y con ella la meta de desarrollo sostenible 4, es un claro punto de partida para la llamada agenda global, pero también incluso en el discurso y entendimiento de la educación por UNESCO y otras organizaciones han existido adaptaciones al debate y a los intereses mundiales, esto con el nombre de Plan de acción para la educación 2030.

Por esta razón que es imperativo revisar la discusión en torno a la educación, no solo en los espacios como las Naciones Unidas o la academia, pues hay todo un espectro que también debe ser tomando en cuenta como los gobiernos, sociedad civil, sociedad mediática. El propósito principal de esto es responder a la pregunta: ¿hay espacio para el cambio en la educación? La idea de espacio no está solo relacionada en términos de espacio físico, pero más bien a la conceptualización de educación como un modelo que refleja unidad entre teoría y práctica en un dialogo interconectado con diferentes actores, manteniendo abierto los canales para escuchar y ser escuchado.

Elsewhere in Europe, notably in Scandinavia, Netherlands and Germany, and also 
in Japan, there is evidence from the 1970s onwards of approaches towards education that promote a more international outlook, under themes such as education for international understanding or intercultural learning. In some countries there was a strong influence from UNESCO, in others from the increased role of the European Commission or in the case of Japan a conscious move from its imperial past to a more outward looking view of the world (Bourn, 2012, p. 26)

Por lo tanto, la construcción del cambio en el espacio debe partir de la premisa de que hay diferentes puntos de partida y finales diferentes. El principal problema en torno a la reconceptualización de la educación es el mantener el enfoque paternalista de alguien que tiene razón y alguien que está equivocado, más bien se debe entender desde la participación a un nivel comunitario que abrace la educación como una práctica de humanismo y visión crítica.

Thus, there is a lack of internal critique in the field and of dialogue with other disciplines where debates about globalisation, identity and global politics and development are in full swing. The area seems to be isolated and undertheorised, as McCollum pointed out in 1996: 'The development education debate (...) remains at a superficial level precisely because there is little discussion of the theory implicit in the practice. (Andreotti, 2006)

El objetivo no es criticar la comprensión real de la educación para el desarrollo, la educación sostenible, el aprendizaje global o la educación, sino ver cuánto han cambiado las dinámicas en torno a la educación. Pensar que hay espacio para el cambio implica aplicar la alfabetización crítica al sistema actual (aplicar la pregunta a nivel micro). La educación de alguna manera ha sido estandarizada en varias conceptualizaciones donde en todas ellas juega un papel importante la instrumentalización del "norte" enfocándola como una herramienta que desempeña un papel importante que puede reducir las brechas de desigualdad y poder entre el norte y el sur.

Sin embargo, la necesidad de conceptualizar el norte y, por lo tanto, el sur crea la primera dicotomía sobre el papel de la educación. Se ha argumentado que, como parte de la discusión, la educación en términos de desarrollo debe entenderse como aprendizaje global y hay una fuerte razón para eso. El término desarrollo tiene implícito un tipo de proceso tan experimentado que debe solucionarse. Por lo tanto, hablar de educación para el desarrollo es hablar de educación para los subdesarrollados, el proceso de esta dicotomía simple es parte de los roles de poder o las relaciones entre el norte y el sur; la posición benevolente desde la cual la educación se proporciona como ayuda para el otro, al que se considera menos y necesita "ayuda" llegar a la idea de desarrollo.

Esta parte del debate es aún más clara cuando se pone en contraste con los objetivos del Milenio y más tarde con los objetivos sostenibles. Por otro lado, el aprendizaje global o el aprendizaje sostenible implica una mejor interacción entre los sujetos. Existe la oportunidad de aprender de la experiencia del sur global, por ejemplo, la conexión con la naturaleza y la necesidad sostenible del conocimiento indígena como un sistema válido desde donde el concepto de "desarrollo" podría tener una mejor interpretación. Un claro ejemplo de esto es la idea de sumak kawsay -buen vivir-, pero este no es el espacio para expandir esa idea. 
Volviendo al debate, el cambio de un concepto a otro y, al mismo tiempo, la falta de éxito de la educación para el desarrollo en el hemisferio sur tal vez se basa en el simple hecho de que las soluciones provenientes del Norte todavía no están bien conectadas con el sur; todavía sigue habiendo una falta de conexión intercultural en lugar de multicultural. El problema es más complejo que solo aplicar los modelos de los países en desarrollo y ver si funcionan en África o Sudamérica, debe haber un diálogo multilingüe e interesado en comprender al otro, no solo para llevar al otro a lo que se conceptualiza como bueno, sino sentir y ser parte del espacio del otro.

Therefore, there are (at least) four different possibilities for thinking and action. The first possibility is 'think as I do, do as I say, there is only one right answer'. The example from development education I use is a quote from a teacher: 'I teach my students that people in poorer countries lack technology, education and proper work habits. I make sure my students understand that we have a moral obligation to help them by providing assistance through charity and expertise.' The second possibility is 'think for yourself and choose responsibly what to do, but there is only one right answer', which is illustrated in the quote: 'I teach my students that they need to be critical thinkers - to separate facts from opinions and to search for impartial, objective information to construct their arguments. I believe rational and scientific reasoning is the only way to achieve a just and prosperous society. (Andreotti, 2014, p. 18)

Una prueba de una mejor conexión, incluso teniendo en cuenta la falta de recursos, es la aplicación de mejores programas entre el sur global. Hay muchos ejemplos que deben tenerse en cuenta en la llamada cooperación Sur-Sur. Existe una narrativa entre las diferentes regiones del mundo que, debido a las similitudes (Colonización y estructuras coloniales) hablan entre sí de una mejor manera, no hay soluciones simples, pero al menos hay un mejor esfuerzo para entender al otro.

"Within the wisdom language of a collective Mestiza/o Body, curriculum lives and breathes to defy and ultimately heal a colonizing curriculum of " illegal histories, rape, murder, slavery, bad pronunciation of the names of streets, rivers, towns, the loss of our names entirely" (Sosa-Provencio, 2018, p. 98)

Por lo tanto, la falta de éxito también se encuentra en los espacios ambiguos donde se desarrolla la discusión de la educación, la teoría y la práctica no se relacionan con los espacios reales y las necesidades reales, en un sentido más claro lo qué desarrollo o medios sustentables significa en la academia no esta tan cerca de lo que significa en pequeñas comunidades en la selva amazónica o en pequeños países insulares de Oceanía. El espacio de la discusión en torno a la educación está lleno de narrativas incompletas.

A partir de este punto, el re-pensar la educación es importante y para eso es necesario aprender a desaprender, aprender qué aprender y aprender cómo escuchar. El problema del cambio en la educación es que nada ha cambiado en la base de la educación, todavía existe la idea de que hay un buen sistema y un mal sistema de educación, también existe una fuerte relación entre el lenguaje y la educación, es por esto que existe la firme creencia que algunos 
idiomas son más importantes que otros, y que con algunos idiomas la gente logrará más que con otros y esto es solo el resultado de la falta de espacio en el cambio de la educación.

Debe haber una reapropiación del espacio, no hay una mala educación, pero en cuanto a qué sistema y como se aplica debe ser discutido, porque lo que se enseña en la jungla de Ecuador es crucial en términos de identidad cultural, aprendizaje de la vida y raíces, y es este proceso de aprendizaje global pero debe poner al resto del mundo en una clara percepción de no solo una educación para el desarrollo, sino un sistema de educación múltiple.

Entonces, el diálogo debe tener lugar en los diferentes espacios, el espacio indígena, el espacio intercultural, el espacio progresivo, el espacio tecnológico y otros. la conectividad de estos espacios en diferentes niveles y situaciones podría dar lugar a un verdadero aprendizaje global y, por lo tanto, a una respuesta positiva a la pregunta más importante. ¿Hay espacio para el cambio en la educación? Y si la respuesta se pierde en teoría, puede que algo esté mal.

\section{Comentarios finales: Educación de Occidente, el resto y nuevas posibilidades}

Andreotti (2014) presentó en su alfabetización crítica preguntas importantes como: por qué se creó la educación para el desarrollo, quién creó y con qué propósito, dónde se centró con las regiones, cómo y por qué se presenta a los gobiernos locales. El objetivo principal de esta pregunta es volver a enmarcarnos en el debate no solo de la educación para el desarrollo sino en la continuidad de la misma. Tal vez haya un cambio en la terminología, pero estas preguntas son un punto de partida sólido para cualquier investigación en términos de cambio estructural.

Ha generado una dicotomía, la dualidad en cuanto a lo que representa la noción de educación para el desarrollo de occidente y lo que sería para el denominado "resto", una categoría en la que América Latina también ha sido enmarcada en varias ocasiones (Hall, 1992). Aquí la importancia de poder ofrecer categorías como mecanismo de opresión o control (Freire). La categorización otorgada a América Latina y África impulsa la necesidad de estudios no solo pos-coloniales sino descoloniales, tomando en cuenta su propia historia. Bajo esta premisa sostiene la idea de relación entre ambas regiones más allá de la colonización en términos de sistemas educativos (Rodney, 1973)

The concept or idea of "the West" can be seen to function in the following ways: First, it allows us to characterize and classify societies into different categories... Secondly, it is an image, or set of images. It condenses a number of different characteristics into one picture...Thirdly, it provides a standard or model of comparison. It allows us to compare to what extent different societies resemble, or differ from, one another... Fourthly, it provides criteria of evaluation against which other societies are ranked and around which powerful positive and negative feelings cluster... the West itself was produced by certain historical processes operating in a particular place in unique (and perhaps unrepeatable) historical circumstances... (Hall, 1992, p. 277) 
La protesta social desde un punto de vista cultural e histórico da los argumentos necesarios para la construcción del pensamiento crítico alrededor de los fenómenos de cada región. Enmarcar la realidad política, la protesta social y la emancipación como principios universales es caer en la lógica marxista que no permite ubicar estos fenómenos como principios que no son universales y que tienen sus propias características." En lugar de este enfoque limitante, el análisis debe partir de la realidad política y ubicar la educación en esa realidad en lugar de suponer que la educación es un fenómeno universal con una política fija" (Branch \& Zachariah, 2015, p.7).

The Papua New Guinea narrative illustrates the gamut of a comparative education research agenda as it demonstrates the translations and dialectics of global agendas in a postcolonial setting. International goals for education are designed to address real educational issues; however, the contextual complexities that impede successful translation of global imperatives are deeply entrenched with ongoing uncritical acceptance of educational priorities and models, dependency on foreign finance and conceptualization for leading educational change (Mohok McLaughlin, 2017, pp. 217 - 218)

La construcción o búsqueda de una identidad basada en el otro (otredad) o en la diferenciación del otro, del colonizador, nos permite observar en una primera instancia ciertas pinceladas o similitudes en la narrativa de ambos continentes, tomemos el ejemplo de Oceanía y la relación entre lo que se enseñó y lo que se necesitaba, esto juega un papel muy importante en la vida de las diferentes comunidades. En la misma línea, pero con resultados diferentes, la agenda impositiva del colonizador para controlar el sistema educativo del "mestizo" tampoco muestra el impacto creativo de la educación sino el impacto destructivo de la pedagogía sin un sentido de construcción sino de imposición.

"In the lives of Indigenous and Indigenous-heritage Mexicana/o youth, the imposed curriculum that ordered colonial schooling has been one of the strongest weapons in the cultural and political conquest of the Americas (Gonzales-Berry, 2000 ; González, 1999 ; Kleyn, 2010 ; Lomawaima \& McCarty, 2006 ; San Miguel, 1999 ). Anzaldúa (1990 ) characterizes this imposed infiltration of the colonizers' language, worldview, religion, and knowledge systems as forced cultural penetration" (Anzaldúa, 1990 , p. 143); (Sosa-Provencio, 2018, p. 95)

El papel del educador es dar nacimiento a una nueva sociedad que a través de la implementación de su rol completo para las nuevas generaciones, construya o impulse la necesidad de una educación crítica, lo cual brindara actitudes críticas en los estudiantes, a partir de aquí el proceso de usar nuevos espacios para volver a dar forma a la educación es más factible que en otro tipo de marco. La pregunta al comienzo de este ensayo fue abrir la posibilidad de cambio en el espacio de la educación. Hay varios debates hoy en día en torno a la educación y el papel de la educación, estos debates deben ser adoptados en el sentido de dar nacimiento a sistemas de educación más complejos, dinámicos e integrales.

La contribución especial del educador al nacimiento de la nueva sociedad tendría que ser una educación crítica que podría ayudar a formar actitudes críticas, ya que la conciencia ingenua 
con la que la gente había emergido en el proceso histórico les hacía una presa fácil de la irracionalidad.

Desde un enfoque realista hay estructuras y superestructuras en el sentido de Marx que deben ser superadas, sin embargo, la alfabetización crítica combinada con un mundo más curioso ha acelerado la aparición de muchos movimientos para desafiar esto, en pro de un nuevo sistema dinámico, no solo dinámico, pero más respetuoso y consciente, en un desarrollo más sostenible.

\section{Referencias}

Andreotti, V. (2014). Critical literacy: theories and practices in development education . Policy \& Practice: A Development Education Review , 12 - 32.

Andreotti, V. (2014). Critical literacy: theories and practices in development education. Policy \& Practice: A Development Education Review , 12 - 32.

Andreotti, V. (2006). 'Theory without practice is idle, practice without theory is blind*': the potential contributions of postcolonial theory to development education. The development education Journal , 12 (3), 7-10.

Bourn, D. (2012). Development education: from the margins to the mainstream. $f(x)=$ (Education Global) Research , 1 (1).

Branch, A., \& Zachariah, M. (2015). Africa Uprising: Popular Protest and Political Change. London: Zed Books.

Escobar, A. (1995). The making and unmaking of the third world. princeton: princeton university press.

Freire, P. (2005). Education for critical consciousness. New York: Continuum.

Gerhardt, H. -P. (1994). Paulo Freire. Thinkers on education, 2, 439 - 458.

Hall, S. (1992). The West and the Rest. Discourse and Power. En S. Hall, \& B. Gieben, Formations of modernity (págs. 275 - 331). Cambridge: Polity Press.

Medrick, R. (2013). A Pedagogy for Sustainability Education. Journal of Sustainability Education, 5.

Mohok McLaughlin, J. (2017). "Shifting Boundaries and Education Development Discourses: Implications for Comparative and International Education in Oceania". Annual Review of Comparative and International Education 2017, 205 - 230.

Rodney, W. (1973). How Europe Underdeveloped Africa. (J. Arriola, Trad.) London: London and Tanzanian Publishing House,. 
Sosa-Provencio, A. (2018). Curriculum of the Mestiza/o Body: Living and Learning Through a Corporal Landscape of Resistance and (Re)generation. Diaspora, Indigenous, and Minority Education , 95 - 207. 